\title{
RECOGNIZING MANIFOLDS AMONG GENERALIZED MANIFOLDS
}

\author{
DENNIS C. HASS
}

Abstract. This paper provides various conditions, on the complement of a point in a generalized manifold $M$, which imply that $M$ is a classical topological manifold. Similar characterizations are given for $m$-spheres and 3-cells.

This paper announces a few results in the classic quest for a property which characterizes the topological manifolds among the generalized manifolds.

It is well known, for example, that if a 3-gm $M$ is a product space then it is a manifold. In [1], Raymond showed that the factors are generalized manifolds. Further, Wilder [2] says these factors are manifolds; thus, $M$ is too. Clearly, then a 3-gm which is locally a product space is also a manifold.

Efforts have been made to weaken this hypothesis. In [3] K.W. Kwun and $\mathrm{F}$. Raymond proved that a 3-gm which is locally conical is a manifold. $M$ is locally conical if for all $P$ in $M, P$ has a neighborhood $N$ such that $N-P=E^{1} \times b N$. Our results show that one need not specify the factors in advance. By assuming, only, that $N-P$ is any product space, we can show that $M$ is still a manifold; see Theorem 3.

LEMMA 1. If $M$ is a connected $m$-gm, for $m \geqq 2$ such that for $P$ in $M$, $M-P=A \times B$ is a product space, then each of $M-P, A$, and $B$ is homologically trivial up through dimension $m-2$.

Proof. Proof of these claims is exactly analogous to Chapter 3 of the author's dissertation [4], except for one minor change. We replace the fact that $\pi_{k} A \times B=\pi_{k} A \times \pi_{k} B$ with the Künneth formula and an induction on $k$. It is also still true, that if $B$ is compact, then $M-P=E^{1} \times B$.

Let $M$ be a connected 3-gm.

Theorem 2. For $P$ in $M$, if $M-P$ is a product space, then $M$ is either $S^{3}$ or $E^{3}$.

Received by the editors August 25, 1971.

AMS 1970 subject classifications. Primary 54F65, 55A40, 57B99, 57A15; Secondary 54D45, 57A05, 57A10, 57C99.

Key words and phrases. Topological manifolds, classical manifolds, generalized manifolds, locally conical generalized manifold, spheres, cells.

(c) American Mathematical Society 1972 
Proof. By Lemma 1 , the factorization of $M-P$ is either $E^{1} \times E^{2}$ or $E^{1} \times S^{2}$, respectively.

THEOREM 3. If for each $P$ in $M$, there is an open neighborhood $N$ of $P$ such that $N-P$ is a product space, then $M$ is a classical 3-manifold.

Proof. If $N$ is compact, then $N=M$ and $M=S^{3}$, by Theorem 2. If $N$ is not compact, then $N=E^{3}$ by Theorem 2 .

Now, let $M$ be a connected $m$-gm, for $m \geqq 4$.

THEOREM 4. If for $P$ in $M, M-P$ is a product space, then either $M-P$ is homologically trivial or $M-P$ is $E^{1}$ times a generalized $(m-1)$-sphere.

Proof. By Lemma 1 , each of $M-P=A \times B$, and $A$, and $B$ is $(m-2)$ connected with respect to homology. If neither $A$ nor $B$ is compact, then each is homologically trivial and of course so is $M-P$. If $B$ is compact, then as mentioned in the proof of Lemma 1 , we have $M-P=E^{1} \times B$. Since $B$ is closed and (m-2)-connected (homology) the theorem follows.

THEOREM 5. If for $P$ in $M, M-P$ is a product of (many?) factors each of dimension 2 or less, then $M=S^{m}$.

Proof. In view of Lemma 1, we may assume that none of the factors is compact. According to Theorem 4, each factor is either $E^{1}$ or $E^{2}$.

Next, let $M$ be a compact connected $m$-gm, for $m \geqq 5$.

THEOREM 6. For $P$ in $M$, if $M-P$ is a product of simply-connected (homotopy fundamental group is trivial) PL manifolds $A$ and $B$, then $M=S^{m}$.

Proof. Using Theorem $4, M-P, A$, and $B$ are each $(m-2)$-connected (homotopy this time!). If neither $A$ nor $B$ is compact, then each is contractible.

J. Stallings [5] proved that in this case $M-P=E^{m}$. Finally, let $M$ be a connected $m$-gm, for $m \geqq 6$.

THEOREM 7. If for all $P$ in $M$, there is a neighborhood $N$ of $P$ such that $N-P=A \times B$ is a product of simply-connected manifolds $A$ and $B$, then $M$ is a classical m-manifold.

Proof. By Theorem 4, each of $N-P, A$, and $B$ is $(m-2)$-connected (homotopy!). In light of Theorem 6, we may assume that $B$ is compact. By Lemma $1, N-P=E^{1} \times B$ and $B$ is a homotopy $(m-1)$-sphere. Since $m-1 \geqq 5$ we have $B=S^{m-1}$ by the Poincaré theorem. Thus, $N=E^{m}$ as desired.

That $N-P=A \times B$ inherits the manifold property from $A$ and $B$ is not new; it is new that the homology groups of $A$ and $B$ may be calculated 
and need not be assumed. Note that none of the results here or in [4] relies on the unproven Poincaré conjectures.

ACKNOWLEDGEMENT. I want to thank the Mathematics Department of Randolph-Macon Woman's College for financial support while I studied this topic.

\section{BIBLIOGRAPHY}

1. F. Raymond, Separation and union theorems for generalized manifolds with boundary, Michigan Math. J. 7 (1960), 7-21. MR 22 \#11388.

2. R. L. Wilder, Topology of manifolds, Amer. Math. Soc. Colloq. Publ., vol. 32, Amer. Math. Soc., Providence, R.I., 1949. MR 10, 614.

3. K. W. Kwun and F. Raymond, Generalized cells in generalized manifolds, Proc. Amer. Math. Soc. 11 (1960), 135-139. MR 22 \#7111.

4. D. C. Hass, The ends of a product manifold, Thesis, Michigan State University, East Lansing, Mich., 1970.

5. J. R. Stallings, The piecewise-linear structure of Euclidean space, Proc. Cambridge Philos. Soc. 58 (1962), 481-488. MR 26 \#6945.

Department of Mathematics, Randolph-Macon Woman's College, Lynchburg, VIRGINIA 24504 